Research Paper

\title{
MicroRNA-216b-5p Functions as a Tumor-suppressive RNA by Targeting TPT1 in Pancreatic Cancer Cells
}

\author{
Yu You*, Jiaxin Tan, Yi Gong, Haisu Dai, Haowei Chen, Xuejun Xu, Aigang Yang, Yujun Zhang, Ping Bie ${ }^{\bowtie}$ \\ Department of Hepatobiliary Surgery, Southwest Hospital, Third Military Medical University, Chongqing, 400038, China \\ * These two authors contributed equally to this work \\ $\bowtie$ Corresponding author: Professor Ping Bie, Department of Hepatobiliary Surgery, South Western Hospital, Third Military Medical University, Chongqing, \\ 400038, China. Address: No.30 Gaotanyan Street, Shapingba District, Chongqing, 400038, China. Tel.: +86 2363815300 Fax: +86 23 63815300 E-mail: \\ bieping@medmail.com.cn. \\ (c) Ivyspring International Publisher. This is an open access article distributed under the terms of the Creative Commons Attribution (CC BY-NC) license \\ (https://creativecommons.org/licenses/by-nc/4.0/). See http://ivyspring.com/terms for full terms and conditions.
}

Received: 2016.12.26; Accepted: 2017.05.01; Published: 2017.08.23

\begin{abstract}
MicroRNAs (miRNAs) are increasingly recognized as being involved in pancreatic cancer progression by directly regulating the expression of their targets. In this study, we showed that miR-216b-5p expression was significantly decreased in pancreatic cancer tissues and cell lines. In addition, low miR-216b-5p expression was significantly associated with large tumor size and advanced TNM stage. Meanwhile, both Kaplan-Meier and multivariate survival analysis showed that decreased miR-216b-5p expression was associated with overall survival. miR-216b-5p over-expression repressed pancreatic cancer cell proliferation and induced cell cycle arrest and cell apoptosis in vitro and inhibited tumorigenesis in vivo. The translationally controlled tumor protein (TPT1) was identified as a novel direct target of miR-216b-5p. miR-216b-5p up-regulation suppressed TPTI expression. Moreover, TPTI mRNA expression levels were increased in pancreatic cancer tissues, and were inversely correlated with miR-216b-5p expression. TPT1 down-regulation had similar effects as miR-216b-5p up-regulation on pancreatic cancer cell progression. The restoration of TPT1 reversed the effect of miR-216b-5p on pancreatic cancer cell progression. Furthermore, we found that miR-216b-5p up-regulation suppressed Pim-3, Cyclin BI, $\mathrm{p}$-Bad and $\mathrm{Bcl}-\mathrm{xL}$ protein expression. However, the effect of miR-216b-5p up-regulation was partly reversed by TPT1 up-regulation in vitro. Taken together, our findings suggested that miR-216b-5p functions as a potential tumor suppressor by regulating TPT1 in pancreatic cancer cells, and it may represent a potential therapeutic target for patients with pancreatic cancer.
\end{abstract}

Key words: miR-216b-5p, TPT1, pancreatic cancer, cell cycle.

\section{Introduction}

Pancreatic cancer is a highly lethal malignancy with occult symptoms, rapid development and early metastasis. The 5-year survival rate is less than 5\% [1]. Despite considerable progress made in understanding the pathogenesis of pancreatic cancer, it remains the 10th most often diagnosed malignancy in the world and the 4th leading cause of cancer related deaths in the United States $[2,3]$. Thus, the development of effective therapies for pancreatic cancer is urgently required.

MicroRNAs (miRNAs) are a class of small non-coding RNAs, composed of 18-23 nucleotides (nt) that are involved in the functional regulation of target genes by binding to the 3 '-untranslated regions (3'UTRs) of their target genes [4-6]. Altered miRNA expression has been reported in almost all types of human cancers, including pancreatic cancer [7-9]. miRNAs can function as oncogenes or tumor suppressor genes, involving multiple pathways and cell functions in pancreatic cancer development and progression, such as in the cell cycle, proliferation, apoptosis, invasion and resistance to therapy [10]. For example, miR-21 is significantly up-regulated in pancreatic ductal adenocarcinoma (PDAC) cell lines 
compared with those in non-malignant cells and functions as an oncogene [11]. The over-expression of miR-21 induces cell proliferation, invasion and chemoresistance to gemcitabine [12]. Conversely, miR-615-5p is abnormally down-regulated in PDAC cells and functions as a tumor suppressor in PADC [13]. Recently, miR-216b was found to play pivotal roles in many cancer types [14-17]. In nasopharyngeal carcinoma and hepatocellular carcinoma, miR-216b suppresses tumor growth and invasion [14, 16]. Additionally, in human gastric adenocarcinoma, $\mathrm{miR}-216 \mathrm{~b}$ is down-regulated and inhibits proliferation and cell cycle progression [17]. Moreover, Egeli U et al. [18] found that reduced miR-216b expression was associated with aggressive tumor characteristics and shortened disease-free survival, suggesting the involvement of miR-216b in pancreatic cancer progression. Nevertheless, the role of miR-216b in the regulation of pancreatic cancer progression has not been investigated.

The translationally controlled tumor protein (TPT1) is a highly conserved multifunctional protein. TPT1 is highly expressed in many malignant tumors, including skin squamous cell carcinoma, glioma and prostate cancer, and regulates cell proliferation, invasion, cell cycle and apoptosis [19-25]. In pancreatic cancer, TPT1 expression is up-regulated, and TPT1 down-regulation represses cell proliferation and induces cell cycle arrest and apoptosis [26]. Thus, TPT1 functions as an oncogene and may be an anticancer target in pancreatic cancer.

In the present study, we found that miR-216b-5p was down-regulated in pancreatic cancer tissues and cell lines, repressed pancreatic cancer cell proliferation and induced cell cycle arrest and cell apoptosis by directly targeting TPT1. Our results suggest that miR-216b-5p functions as a tumor suppressor and may be a potential target for pancreatic cancer therapy.

\section{Materials and methods}

\section{Tissue samples and cell culture}

A total of 49 pairs of pancreatic cancer tissues and related cancer-adjacent normal tissues were obtained from patients who underwent surgical resection at the Southwest Hospital, Third Military Medical University. All the tumor tissues confirmed by pathology were preserved in liquid nitrogen. None of the patients had received chemotherapy or radiotherapy. This project was approved by the Ethical Committee of Third Military Medical University, China.

Aspc-1, Bxpc-3, Panc-1, and SW1990 cells were purchased from the American Type Culture
Collection (Manassas, VA, USA), and the normal human pancreatic duct epithelial cells (hTERT-HPNE) were obtained from the Cell Bank Type Culture Collection of the Chinese Academy of Sciences (Shanghai, China). hTERT-HPNE, as well as Aspc-1, Bxpc-3 and SW1990 cells were grown in RPMI-1640 medium (Invitrogen, CA, USA) containing 10\% fetal bovine serum (Gibco, Grand Island, NY, USA). Panc-1 cells were cultured in Dulbecco's modified Eagle's medium (Invitrogen, Grand Island, NY, USA) containing $10 \%$ FBS.

\section{Quantitative reverse transcription-polymerase chain reaction ( $q R T-P C R$ )}

Total RNA from cells or tissue samples was isolated using TRIzol reagent (Invitrogen, Grand Island, NY, USA), according to manufacturer's instructions. The RNA quality and quantity were measured using a NanoDrop ND-1000 spectrophotometer (NanoDrop Technologies, Rockland, DE, USA), and RNA integrity was determined by gel electrophoresis. cDNA was synthesized using M-MLV Reverse Transcriptase (Promega, Madison, WI, USA). These cDNAs were used to analyze the expression of miR-216b-5p and TPT1 using the SYBR Premix Ex Taq kit (TaKaRa, Dalian, China) with a Stratagene Mx3000P real-time PCR system (Agilent Technologies, Santa Clara, CA, USA). For miR-216b-5p expression, the miRNA-specific forward primer (miR-216b-5p Fwd) and universal reverse primer (Rev) were used, and the expression levels were normalized to U6. For TPT1 mRNA expression, paired primers (TPT1 Fwd and TPT1 Rev) were used, and the expression levels were normalized to GAPDH levels. The relative gene expression levels were calculated using the $2^{-\Delta \Delta \mathrm{Ct}}$ method with U6 or GAPDH as an internal control. All the primers were purchased from Sesh-biotech (Shanghai, China) and were listed in Table 1.

\section{Cell transfection}

The miR-216b-5p expression plasmid (miR-216b-5p) and scrambled miRNA expression plasmid (NC) were purchased from GeneCopoeia (Guangzhou, China). The TPT1 expression plasmids were constructed by inserting the TPT1 CDS expression clone into the pcDNA3.1 vector (Sesh-biotech, Shanghai, China). The TPT1 siRNA and negative control siRNA (con) were purchased from GeneChem (Shanghai, China). The sequences for the TPT1 siRNA and negative control siRNA (con) used for the experiments were as follows: TPT1 siRNA: 5' - AAGGUACCGAAAGCACAGU-3' con: 5'-UUCUCCGAACGUGUCACGU-3' 
Table 1. PCR primers

\begin{tabular}{ll}
\hline Primer name & Primer sequence \\
\hline miR-216b-5p RT & $5^{\prime}$-GTCGTATCCAGTGCAGGGTCCGAGGTGCACTGGATACGACTCACATTT-3' \\
miR-216b-5p Fwd & $5^{\prime}$-GGGGAAATCTCTGCAGGCAA-3' \\
U6 RT & $5^{\prime}$-GTCGTATCCAGTGCAGGGTCCGAGGTGCACTGGATACGACAAAATATGGAAC-3' \\
U6 Fwd & $5^{\prime}$-GTGCTCGCTTCGGCAGC-3' \\
Rev & $5^{\prime}$-CAGTGCAGGGTCCGAGGT-3' \\
TPT1 Fwd & $5^{\prime}$ - TATTGGACTACCGTGAGG-3' \\
TPT1 Rev & $5^{\prime}$ - CAAGCAGAAGCCAGTTATG-3' \\
GAPDH Fwd & $5^{\prime}$ - CGGAGTCAACGGATTTGGTCGTAT-3' \\
GAPDH Rev & $5^{\prime}$ - AGCCTTCTCCATGGTGGTGAAGAC-3' \\
wt-TPT1 Fwd & $5^{\prime}$ - TAGGCGATCGCTCGAGCAAATGTGGCAATTATT-3' \\
wt-TPT1 Rev & $5^{\prime}$ - AATTCCCGGGCTCGAGTTATCAGTGTTCTCAAGTTCC-3' \\
mut1-TPT1 Fwd & $5^{\prime}$-ACCAAGAGAATAAAAAGAGACTCATTCA-3' \\
mut1-TPT1 Rev & $5^{\prime}$-CTCTTTTTATTCTCTTGGTTCCAGTTAACTACTTCCGTT-3' \\
mut2-TPT1 Fwd & $5^{\prime}$-GAGGGTGCAGTGAGCTGTGATCATACCA-3' \\
mut2-TPT1 Rev & $5^{\prime}$-AGCTCACTGCACCCTCCGGACTCAAGCCATCCTCTCA-3' \\
\hline
\end{tabular}

Bxpc-3 and SW1990 cells were transfected using Lipofectamine 2000 (Invitrogen, Grand Island, NY, USA) in 6-well or 96-well plates and then were further incubated for $24 \mathrm{~h}$ or $48 \mathrm{~h}$, according to the manufacturer's instructions.

\section{Cell proliferation assay}

Cell proliferation was determined by the Cell Counting Kit-8 kit (CCK-8) (Beyotime, Hangzhou, China). Bxpc-3 and SW1990 cells were transfected with the scrambled miRNA expression plasmid (NC), miR-216b-5p expression plasmid (miR-216b-5p), negative control siRNA (con) and TPT1 siRNA alone or the miR-216b-5p expression plasmid with the TPT1 expression plasmid and were seeded onto 96-well plates $\left(4 \times 10^{3}\right.$ cells per well) and further incubated for $1,2,3$, and $4 \mathrm{~d}$. Next, the absorbance at $450 \mathrm{~nm}$ was measured with a microplate reader (Thermo Scientific, Waltham, MA, USA). All the assays were performed in triplicate.

\section{Cell cycle analysis}

For the cell cycle analysis, Bxpc-3 and SW1990 cells were plated in 6-well plates at a final concentration of $5 \times 10^{5}$ cells/well. After $24 \mathrm{~h}$, the cells were transfected and were further incubated for $48 \mathrm{~h}$. Next, the cells were washed in PBS and were fixed with $75 \%$ cold ethanol overnight at $-20^{\circ} \mathrm{C}$, treated with RNaseA (Sigma-Aldrich, St. Louis, MO, USA), and then stained with $50 \mu \mathrm{g} / \mathrm{ml}$ propidium iodide (Sigma-Aldrich, St. Louis, MO, USA) for $10 \mathrm{~min}$. After incubation, the cells were subjected to flow cytometry analysis using a FACSCalibur flow cytometer (BD Biosciences, San Diego, CA, USA). All the assays were performed in triplicate.

\section{Cell apoptosis assay}

For the cell apoptosis assay, Bxpc-3 and SW1990 cells were plated in 6-well plates at a final concentration of $6 \times 10^{5}$ cells/well. After $24 \mathrm{~h}$, the cells were transfected and further incubated for $48 \mathrm{~h}$. After $48 \mathrm{~h}$ of incubation, the cells were washed twice with cold PBS and stained using the Annexin V/PI detection kit (BD Biosciences, San Diego, CA, USA) following the manufacturer's protocol. All the experiments were performed in triplicate.

\section{In vivo tumor growth assay}

Four-week-old BALB/c nude mice were purchased from the Animal Center of the Chinese Academy of Science (Shanghai, China) and were maintained under pathogen-free conditions. For the tumor growth assay, SW1990 cells were transfected with the scrambled miRNA expression plasmid (NC) or miR-216b-5p expression plasmid (miR-216b-5p) and were selected with $6 \mu \mathrm{g} / \mathrm{ml}$ puromycin (Thermo Scientific, Waltham, MA, USA) for two weeks. Then, SW1990 cells $\left(5 \times 10^{6}\right.$ cells in $100 \mu$ of PBS) with stable overexpression of the scrambled miRNA (NC) or miR-216b-5p were subcutaneously injected into nude mice to establish a xenograft. The tumor volume was determined every week and was calculated using the following formula: tumor volume $=$ (length $\times$ width $\left.^{2}\right) / 2$. The mice were sacrificed 5 weeks after injection.

\section{Western blot analysis}

Transfected cells were harvested and lysed in RIPA buffer (Thermo Scientific, Rockford, IL, USA) containing protease inhibitors. The protein concentration was measured using a BCA protein assay (Beyotime, Jiangsu, China). The protein extracts were separated via SDS-PAGE and then transferred to PVDF membranes (Millipore, Billerica, MA, USA). The membranes were blocked with 5\% nonfat powdered milk in PBS and then incubated with primary antibodies at $4^{\circ} \mathrm{C}$ overnight. Rabbit polyclonal antibody against human TPT1, Pim-3 and Cyclin B1, as well as rabbit monoclonal antibodies 
against human p-Bad (Ser112), Bad and Bcl-xL, was purchased from Abcam (Cambridge, MA, USA). The membranes were then washed extensively with TBST and incubated with the secondary antibodies for $1 \mathrm{~h}$ at room temperature. The signal was detected using an enhanced chemiluminescence system (Thermo Scientific, Rockford, IL, USA). The results were normalized to GAPDH and expressed as relative densities.

\section{Luciferase reporter assay}

The wild-type 3'UTR of TPT1 (3915 bp) containing the two putative miR-216b-5p binding sites (Site 1:841-849 and Site 2: 3355-3361) was amplified by PCR using the primer pair wt-TPT1 Fwd and wt-TPT1 Rev from the genomic DNA of SW1990 cells. The PCR product was cloned into the downstream of the luciferase gene in the psiCHECK-2 vector (Promega, Madison, WI, USA). The mutations of the miR-216b-5p putative binding sites in TPT1 3'UTR were also synthesized by PCR. All the vectors were identified by DNA sequencing. All the primers were purchased from Sesh-biotech (Shanghai, China) and listed in Table 1.

Bxpc-3 and SW1990 cells were cotransfected with the TPT1 3'UTR luciferase expression plasmid and the miR-216b-5p expression plasmid or NC using Lipofectamine 2000 (Invitrogen, Grand Island, NY, USA). After $48 \mathrm{~h}$ of transfection, the luciferase activity was determined using a dual-luciferase reporter assay system (Promega, Madison, WI, USA) according to the manufacturer's instructions. The firefly luciferase activity was normalized to Renilla activity.

\section{Statistical analysis}

The data were presented as the means \pm S.D. from at least 3 independent experiments and were analyzed using SPSS17.0 statistical software (IBM
Corporation, Armonk, NY, USA). The difference among the groups in the Western blot, proliferation, cell cycle and apoptosis assays were determined by Student's t-test or one-way ANOVA. The association between miR-216b-5p and TPT1 in pancreatic cancer tissues was calculated using the Spearman's correlation coefficient. The relationship between miR-216b-5p expression and clinicopathologic features of pancreatic cancer was analyzed using the Pearson $x^{2}$ test. Kaplan-Meier method was used to calculate overall survival of 2 patient groups, and differences were analyzed by log-rank test. Univariate Cox regression was performed on each clinical covariate to examine its influence on patient overall survival. Final multivariate models were based on step-wise addition. A $P$ value $<0.05$ was considered to be statistically significant.

\section{Results}

\section{miR-216b-5p expression is down-regulated in pancreatic cancer tissues and cell lines}

To measure the expression of miR-216b-5p in pancreatic cancer tissues, we analyzed miR-216b-5p expression in 49 pairs of human pancreatic cancer tissues and related cancer-adjacent normal tissues by qRT-PCR. Compared with cancer-adjacent normal tissues, miR-216b-5p expression was significantly decreased in pancreatic cancer tissues (Figure 1A; 0.41 \pm 0.20 vs. $0.66 \pm 0.31, \quad P<0.01)$. Additionally, miR-216b-5p expression was analyzed in pancreatic cancer cell lines (Aspc-1, Bxpc-3, Panc-1, and SW1990) and normal human pancreatic duct epithelial cell line (hTERT-HPNE). As shown in Figure 1B, the expression of miR-216b-5p was significantly down-regulated in pancreatic cancer cell lines compared with that of hTERT-HPNE $(P<0.01)$.
A

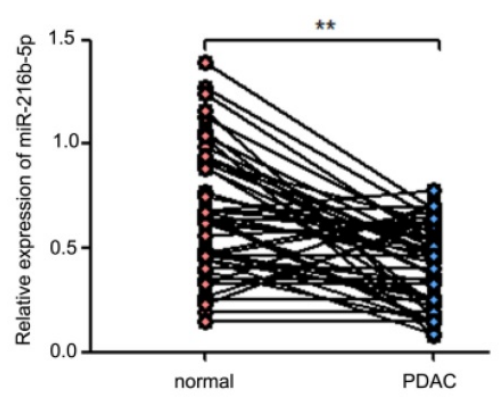

B

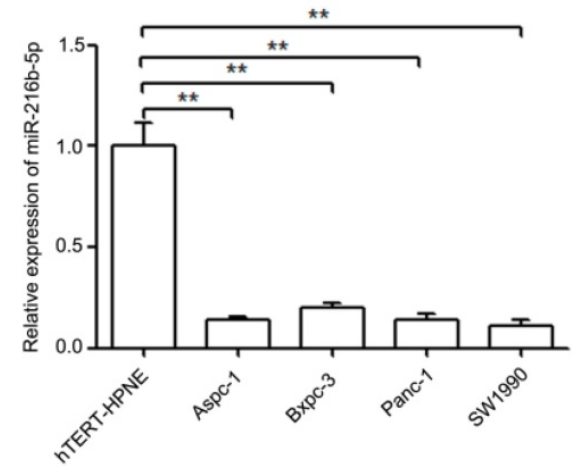

C

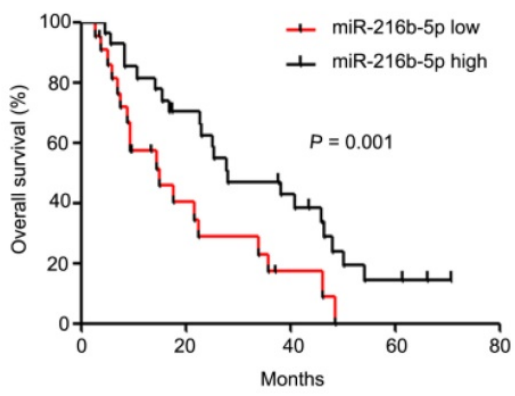

Figure 1. miR-216b-5p expression in pancreatic cancer tissues and cell lines and its relevance to overall survival. (A) The expression levels of miR-216b-5p were analyzed by qRT-PCR in 49 pairs of human pancreatic cancer tissues and related cancer-adjacent normal tissues. U6 was used as a loading control. (B) The expression levels of miR-216b-5p were analyzed by qRT-PCR in four pancreatic cancer cell lines (Aapc-1, Bxpc-3, Panc-1 and SW1990) and one normal human pancreatic duct epithelial cell line (hTERT-HPNE). U6 was used as a loading control. (C) Kaplan-Meier curves for overall survival analysis by miR-216b-5p expression in patients with pancreatic cancer. $P$ value was obtained by a log-rank test. $* *, P<0.01$. 


\section{MiR-216b-5p down-regulation is associated with poorer prognosis in patients with pancreatic cancer}

To determine whether the miR-216b-5p expression level was associated with clinical characteristics and overall survival of patients, a follow-up study was conducted. Our results showed that low miR-216b-5p expression was significantly associated with large tumor size $(P=0.013)$ and advanced TNM stage $(P=0.020)$, but not with patients' age $(P=0.617)$, gender $(P=0.656)$, tumor differentiation $(P=0.287)$, lymph node metastasis $(P=$ $0.097)$, and distant metastasis $(P=0.078)$ (Table 2$)$. Furthermore, Kaplan-Meier survival analysis result showed that patients of pancreatic cancer with low levels of miR-216b-5p expression had a significantly shorter overall survival $(P=0.001)$ (Figure $1 C)$. Meanwhile, multivariate survival analysis showed that distant metastasis and miR-216b-5p were associated with overall survival (Table 3 ). These results indicate that decreased miR-216b-5p expression predicts poorer prognosis in patients with pancreatic cancer.

\section{miR-216b-5p up-regulation represses cell proliferation in vitro and tumorigenicity in vivo and induces cell cycle arrest and apoptosis}

To determine the effect of miR-216b-5p on pancreatic cancer progression, the miR-216b-5p expression plasmid (miR-216b-5p) and scrambled miRNA expression plasmid (NC) were transfected into Bxpc-3 and SW1990 cells, respectively. The miR-216b-5p expression in transfected cells was significantly increased (Figure 2A). Cell proliferation was measured using CCK-8 assays in vitro, and we found that miR-216b-5p up-regulation repressed pancreatic cancer cell proliferation (Figure 2B). Cell cycle analysis using flow cytometry showed that miR-216b-5p up-regulation induced cell cycle arrest in both Bxpc-3 and SW1990 cells (Figure 2C). As shown in Figure 2C, the cell populations in the G1 phase and G2 phase were decreased, and the cell population in the $S$ phase was increased in cells transfected with the miR-216b-5p expression plasmid. Additionally, apoptosis assays showed that miR-216b-5p up-regulation induced pancreatic cancer cell apoptosis (Figure 2D). To investigate the function of miR-216b-5p in pancreatic cancer carcinogenesis, we subcutaneously inoculated SW1990-miR-216b-5p cells and SW1990-NC cells into nude mice in vivo. As shown in Figure 2E, cells transfected with miR-216b-5p generated smaller tumors than cells transfected with NC.

Table 2. Correlations between miR-216b-5p expression and clinicopathological characteristics of patients with pancreatic cancer

\begin{tabular}{|c|c|c|c|}
\hline \multirow{2}{*}{ Clinical factor } & \multicolumn{2}{|c|}{ miR-216b-5p expression } & \multirow{2}{*}{$P$ value } \\
\hline & $\begin{array}{l}\text { Low expression } \\
(\mathrm{n}=22)\end{array}$ & $\begin{array}{l}\text { High } \\
\text { expression } \\
(\mathrm{n}=27)\end{array}$ & \\
\hline \multicolumn{4}{|l|}{ Age(y) } \\
\hline$<60$ & 14 & 19 & 0.617 \\
\hline$\geq 60$ & 8 & 8 & \\
\hline \multicolumn{4}{|l|}{ Gender } \\
\hline Male & 12 & 13 & 0.656 \\
\hline Female & 10 & 14 & \\
\hline \multicolumn{4}{|c|}{ Tumor size (cm) } \\
\hline$<2$ & 6 & 17 & $0.013^{*}$ \\
\hline$\geq 2$ & 16 & 10 & \\
\hline \multicolumn{4}{|c|}{ Tumor differentiation } \\
\hline Well & 8 & 14 & 0.278 \\
\hline Poor & 14 & 13 & \\
\hline \multicolumn{4}{|l|}{ TNM stage } \\
\hline I+II & 5 & 15 & $0.020^{*}$ \\
\hline III+IV & 17 & 12 & \\
\hline \multicolumn{4}{|c|}{ Lymph node metastasis } \\
\hline Negative & 7 & 15 & 0.097 \\
\hline Positive & 15 & 12 & \\
\hline \multicolumn{4}{|c|}{ Distant metastasis(M) status } \\
\hline M0 & 10 & 19 & 0.078 \\
\hline M1 & 12 & 8 & \\
\hline
\end{tabular}

*Statistically significant $(\mathrm{P}<0.05)$

Table 3. Cox proportional hazard models for prognostic factors

\begin{tabular}{|c|c|c|c|c|}
\hline & \multicolumn{2}{|l|}{ Univariate analysis } & \multicolumn{2}{|l|}{ Multivariate analysis } \\
\hline & $\mathrm{HR}(95 \% \mathrm{CI})$ & $P$ value & $\mathrm{HR}(95 \% \mathrm{CI})$ & $P$ value \\
\hline Age $(\geq 60$ vs. $<60)$ & $1.045(0.541-2.021)$ & 0.895 & & \\
\hline Gender (female vs. male) & $1.236(0.655-2.332)$ & 0.512 & & \\
\hline Tumor size $(\geq 2$ vs. $<2)$ & $2.064(1.065-4.001)$ & $0.032^{*}$ & $1.606(0.692-3.725)$ & 0.270 \\
\hline Tumor differentiation (Poor vs. Well ) & $2.168(1.125-4.177)$ & $0.021^{*}$ & $1.399(0.660-2.966)$ & 0.382 \\
\hline TNM stage (III+IV vs. I+II) & $2.435(1.216-4.872)$ & $0.012^{* *}$ & $1.888(0.785-4.541)$ & 0.156 \\
\hline Lymph node metastasis (Positive vs. Negative) & $2.477(1.278-4.801)$ & $0.007^{* *}$ & $1.721(0.765-3.868)$ & 0.189 \\
\hline Distant metastasis (M1 vs. M0) & $4.295(2.113-8.734)$ & $0.000^{* *}$ & $3.527(1.469-8.471)$ & $0.005^{* *}$ \\
\hline MiR-216b-5p (low vs. high) & $3.277(1.595-6.732)$ & $0.001^{* *}$ & $2.243(1.030-4.886)$ & $0.042^{*}$ \\
\hline
\end{tabular}

*Statistically significant $(\mathrm{P}<0.05)$, ** Statistically significant $(\mathrm{P}<0.01)$. 
A

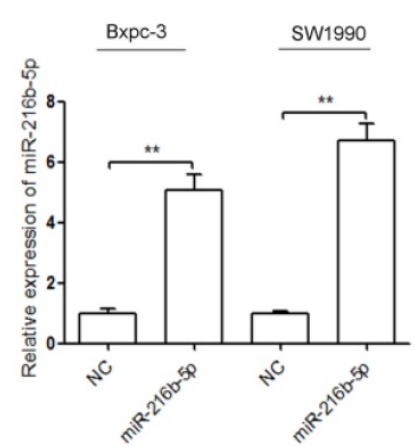

B

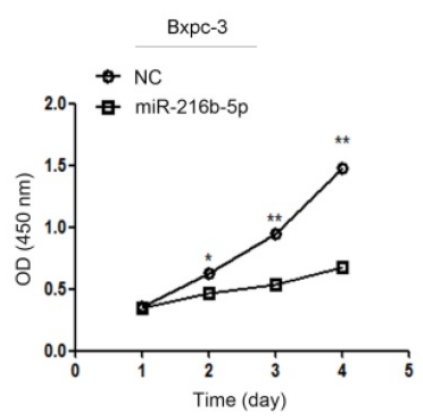

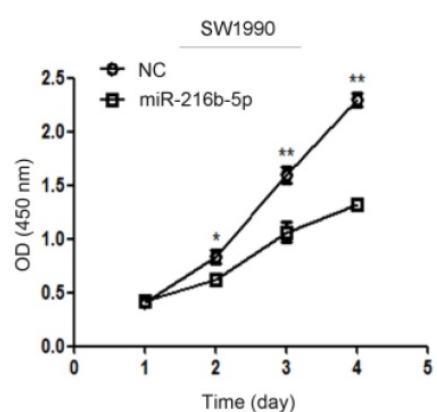

C
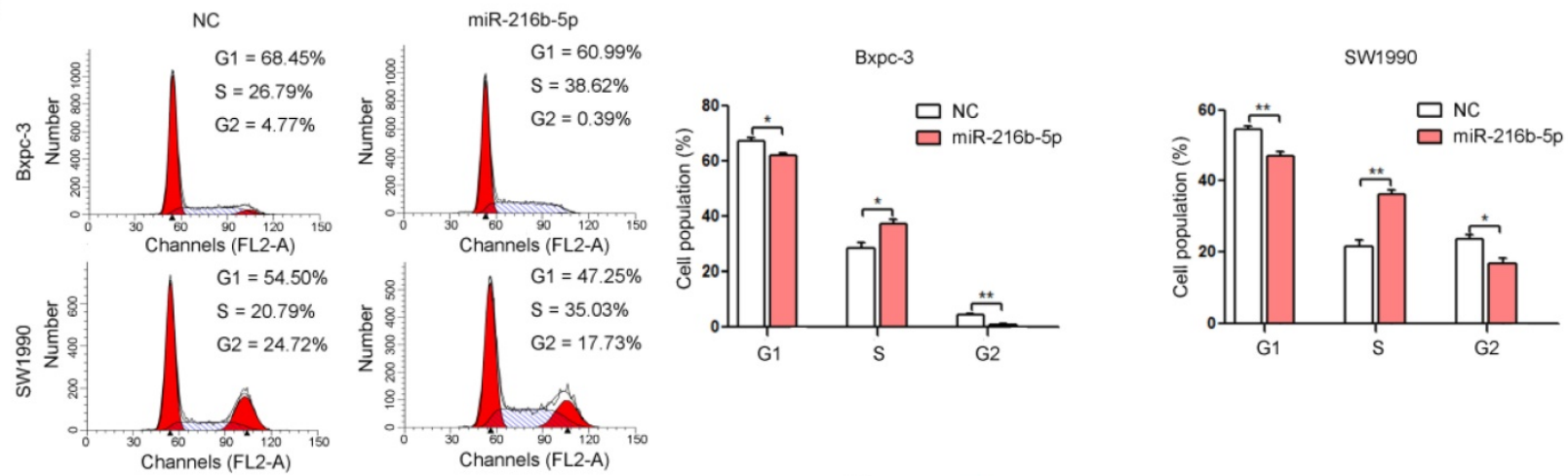

D
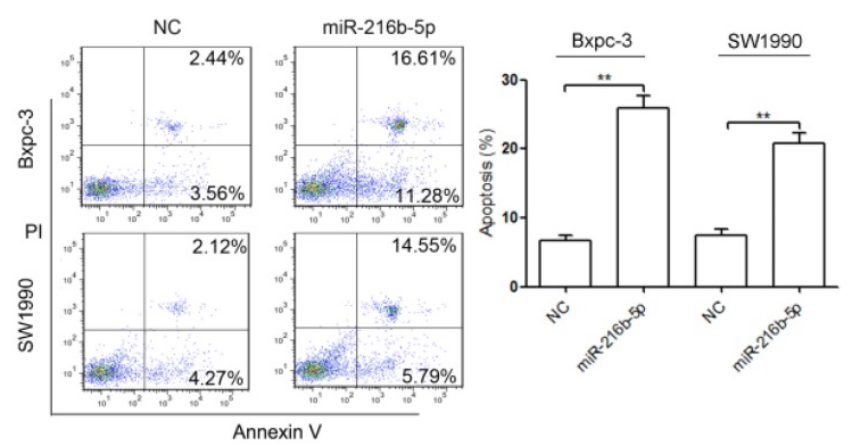

E
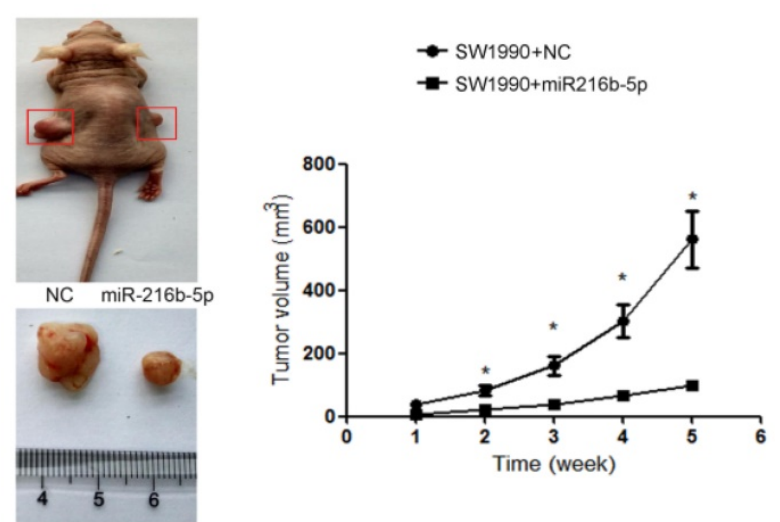

Figure 2. miR-216b-5p up-regulation represses proliferation and induces cell cycle arrest and apoptosis in pancreatic cancer cells. (A) The miR-216b-5p expression levels were detected by qRT-PCR in Bxpc-3 and SW1990 cells transfected with the miR-216b-5p expression plasmid (miR-216b-5p) or scrambled miRNA expression plasmid (NC). U6 was used as a loading control. (B-D) Bxpc-3 and SW1990 cells were transfected with the miR-216b-5p expression plasmid (miR-216b-5p) or scrambled miRNA expression plasmid (NC). Then, the cell proliferation (B), cell cycle (C), and cell apoptosis (D) were analyzed by the CCK-8 assay and flow cytometry, respectively. (E) Growth curve of tumor volumes in all the mice. *, $P<0.05 ; * *, P<0.01$.

\section{TPT1 is characterized as a direct target of miR-216b-5p and is inversely correlated with miR-216b-5p expression in pancreatic cancer}

To identify the potential effectors of miR-216b-5p on pancreatic cancer progression, miR-216b-5p target genes were searched using the microRNA database (www.targetscan.org). TPT1 was identified as a putative target. Sequence alignment demonstrated that there are two putative binding sites for miR-216b-5p in the 3'UTR sequence of TPT1 mRNA, Site 1 and Site 2 (Figure 3A). Western blot results showed that TPT1 protein expression was up-regulated in four pancreatic cancer cell lines compared with that of the hTERT-HPNE (Figure 3B). In addition, miR-216b-5p up-regulation inhibited TPT1 protein expression in Bxpc-3 and SW1990 cells (Figure 3C). Next, to confirm that TPT1 is a direct target of miR-216b-5p, we constructed a luciferase reporter plasmid vector containing the 3'UTR of TPT1 (wt-TPT1) or its mutant version. As shown in Figure $4 \mathrm{D}$, the luciferase activity of miR-216b-5p-transfected cells was significantly reduced compared with that of the NC cells. In addition, site-directed mutagenesis of the miRNA binding sequences in the 3'UTR of TPT1 showed a slightly different trend. Site 1 appeared to be slightly more effective in miR-216b-5p -induced luciferase activity repression because mutation of this 
site significantly reversed a decrease in the luciferase signal (compare Site 1 and Site 2). However, the miR-216b-5p-mediated repression of luciferase activity was abolished by the mutation of both miRNA binding sites (double mutation). Furthermore, we determined the expression of TPT1 in pancreatic cancer tissues by qRT-PCR. As shown in Figure 4E and F, TPT1 mRNA expression was significantly increased in pancreatic cancer tissues compared with that in related cancer-adjacent normal tissues (Figure $3 \mathrm{E} ; 3.69 \pm 1.82$ vs. $1.44 \pm 0.87, P<0.01$ ) and was inversely correlated with miR-216b-5p expression in pancreatic cancer tissues (Figure 3F; $r=-0.495, P<0.01)$. These results suggest that TPT1 is a direct target of miR-216b-5p in pancreatic cancer.

\section{TPT1 down-regulation suppresses proliferation and cell cycle and induces apoptosis in pancreatic cancer cell lines}

Our results showed that miR-216b-5p could repress pancreatic cancer cell progression and directly suppress TPT1 expression. To determine the effect of TPT1 on pancreatic cancer cell lines, the TPT1 siRNA and negative control siRNA (con) were transfected into Bxpc-3 and SW1990 cell lines. As shown in Figure 4A, TPT1 siRNA significantly decreased the expression of TPT1 protein. In addition, we found that TPT1 down-regulation significantly repressed pancreatic cancer cell proliferation (Figure 4B) and induced cell cycle arrest (Figure 4C) and apoptosis (Figure 4D).

A

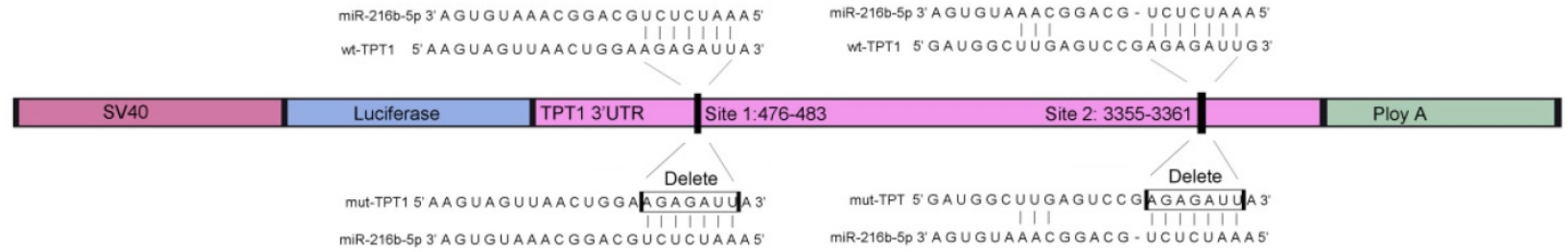

B

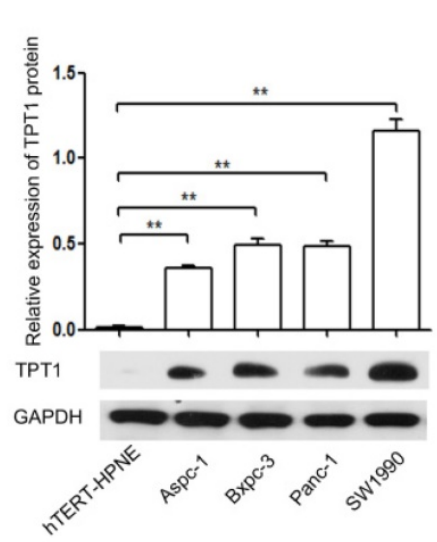

C

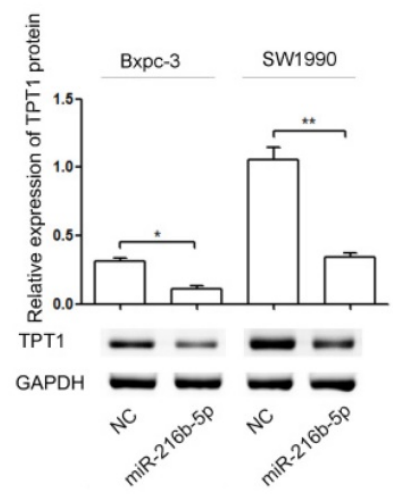

$\mathrm{D}$

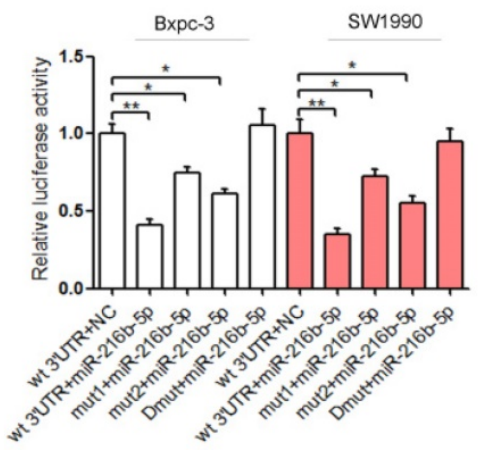

E

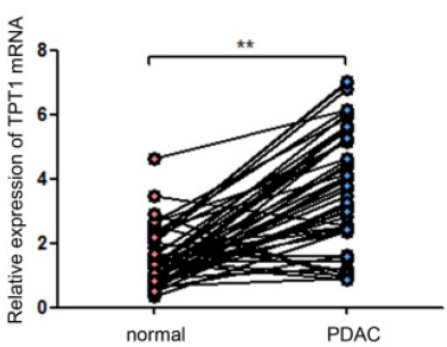

$\mathrm{F}$

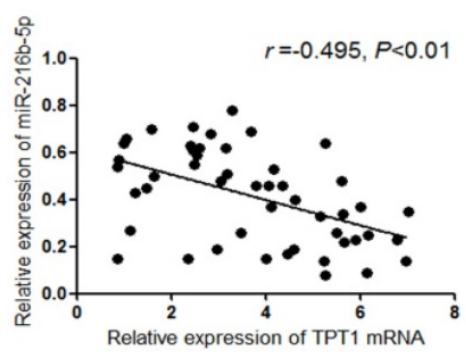

Figure 3. TPT1 is a direct target of miR-216b-5p. (A) The predicted miR-216b-5p binding sequence with the wild-type TPT1 3'UTR (wt-TPT1) and the two mutant TPT1 3'UTR (mut1-TPT1 and mut2-TPT1) constructs used for experiments were indicated. (B) The expression of TPT1 in four pancreatic cancer cell lines and one normal cell line was analyzed by Western blot. GAPDH was used as a loading control. (C) Bxpc-3 and SW1990 cells were transfected with the miR-216b-5p expression plasmid or NC, and then the protein expression levels of TPT1 were detected by Western blot. GAPDH was used as a loading control. (D) Bxpc-3 and SW1990 cells were transfected with the wild-type or mutant TPT1 3'UTR luciferase reporter vector only or co-transfected with the miR-216b-5p expression plasmid or NC for $48 \mathrm{~h}$. The relative luciferase activity was determined by the dual-luciferase assay. Renilla luciferase activity was used as a loading control. (E) The TPT1 mRNA expression levels in 49 pairs of human pancreatic cancer tissues and related cancer-adjacent normal tissues. GAPDH was used as a loading control. (F) An inverse relationship between miR-216b-5p and TPT1 miRNA expression levels was shown in the pancreatic cancer tissues based on Spearman's correlation. The experiments were independently repeated three times. *, $P<0.05 ; * *, P<0.01$. 
A

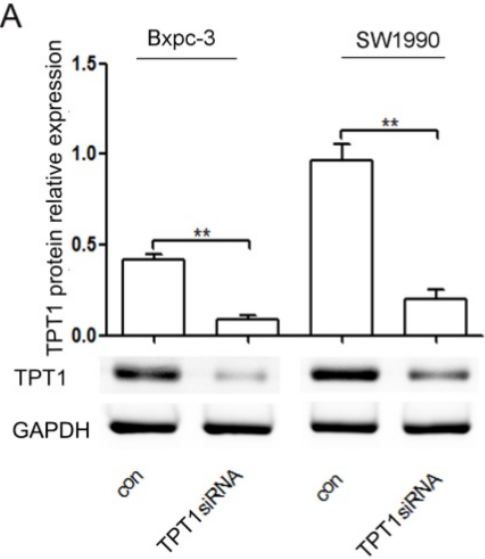

B

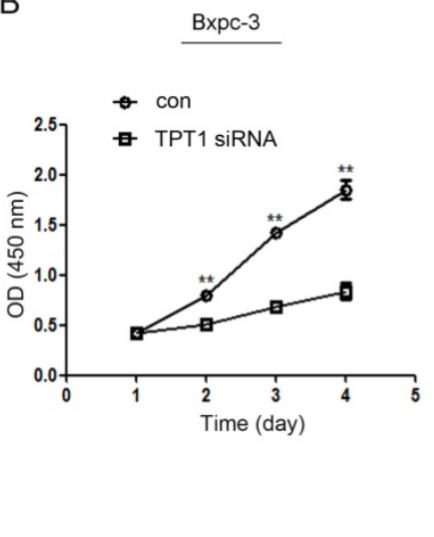

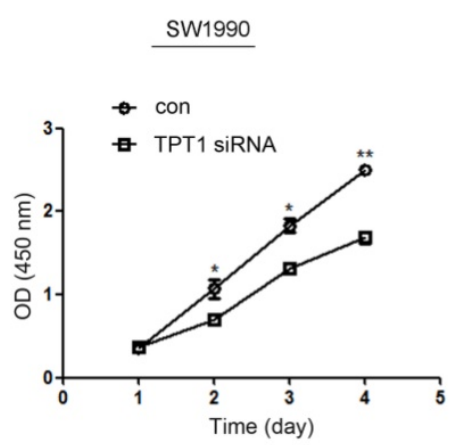

C
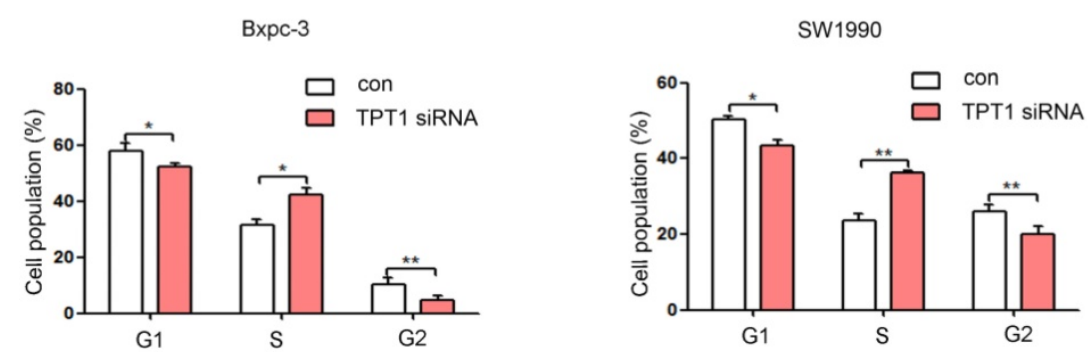

D
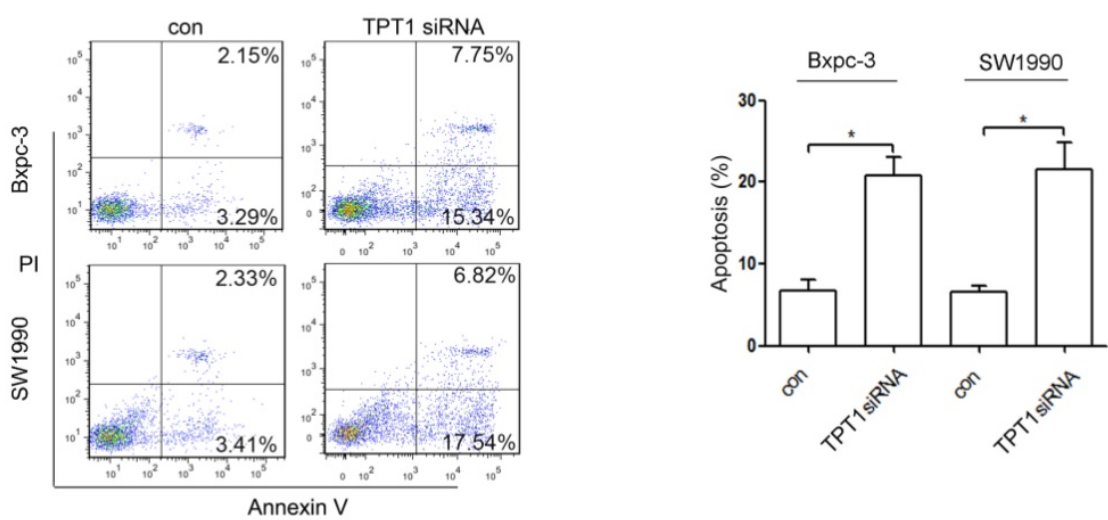

Figure 4. TPT1 down-regulation suppresses proliferation and induces cell cycle arrest and apoptosis in pancreatic cancer cells. (A) The expression levels of TPT1 protein were detected by Western blot in Bxpc-3 and SW1990 cells transfected with the negative control siRNA (con) or TPT1 siRNA. GAPDH was used as a loading control. (B-D) Bxpc-3 and SW1990 cells were transfected with con or TPT1 siRNA. Then, the cell proliferation (B), cell cycle (C), and cell apoptosis (D) were analyzed by the CCK-8 assay and flow cytometry, respectively. The experiments were independently repeated three times. $*, P<0.05 ; * *, P<0.01$.

\section{TPT1 up-regulation partially reverses the suppressive effect of miR-216b-5p on pancreatic cancer cell progression}

To further address that the effect of miR-216b-5p on pancreatic cancer cell progression is mediated by the repression of TPT1, we transfected the cells with an expression construct encoding the entire TPT1 coding sequence that lacked the 3'UTR. As shown in Figure 5A, TPT1 protein expression in TPT1 plasmid-treated cells was significantly up-regulated compared with that in cells transfected with the empty plasmid (pcDNA3.1). In addition, the TPT1 protein levels were restored in the cells cotransfected with miR-216b-5p expression plasmid and TPT1 expression plasmid compared with the protein levels in the cells cotransfected with miR-216b-5p expression plasmid and pcDNA3.1 plasmid (Figure 5B). In functional assays, TPT1 restoration in pancreatic cancer cells transfected with miR-216b-5p expression plasmid rescued the inhibition of cell proliferation and the induction of cell cycle arrest and apoptosis caused by miR-216b-5p up-regulation (Figure 5C, D and E). Collectively, these results demonstrated that the tumor-suppressive effects of miR-216b-5p on pancreatic cancer cells are achieved by directly inhibiting TPT1 expression. 


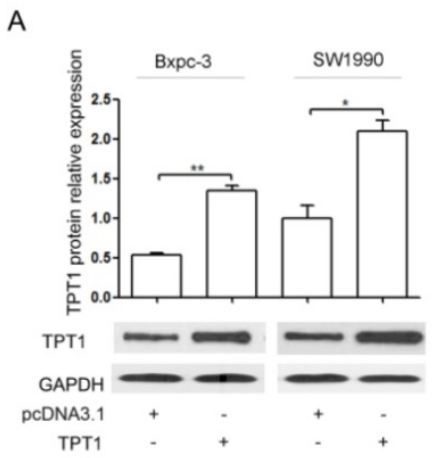

D

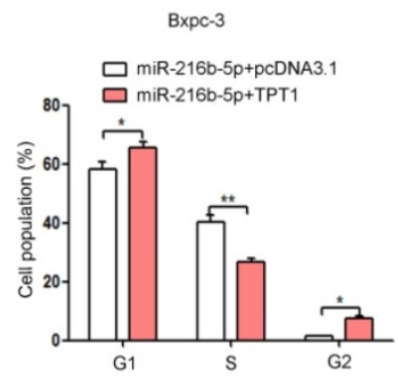

B

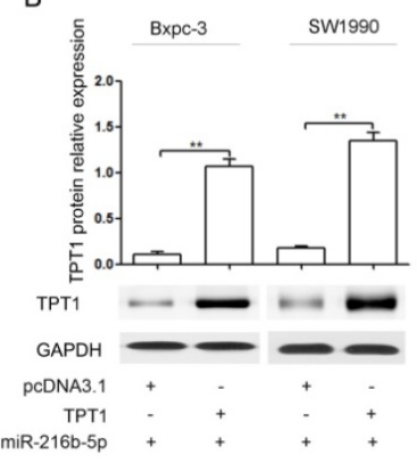

C

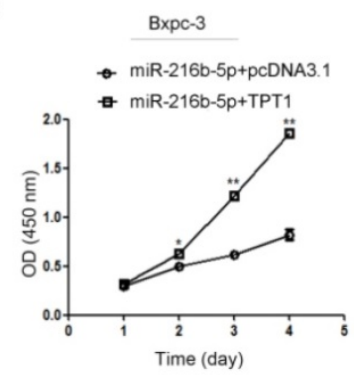

$\mathrm{E}$

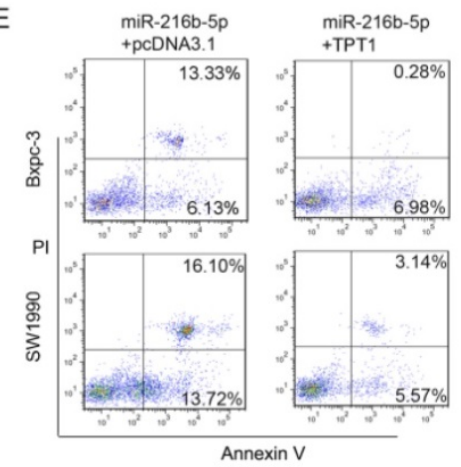

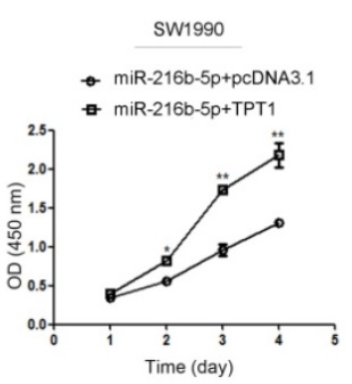

Figure 5. TPT1 restoration partially reverses the effect of miR-216b-5p up-regulation on proliferation, cell cycle, and apoptosis in pancreatic cancer cells. (A) Bxpc-3 and SW1990 cells were transfected with pcDNA3.1 or TPT1 expression plasmid (TPT1). The TPT1 protein expression levels were determined by Western blot. GAPDH was used as a loading control. (B) Bxpc-3 and SW1990 cells were transfected with pcDNA3.1 or TPT1 expression plasmid (TPT1) co-transfected with miR-216b-5p expression plasmid. Western blot was used to confirm the TPT1 protein expression. GAPDH was used as a loading control. (C-E) Bxpc-3 and SW1990 cells were transfected with pcDNA3.1 or TPT1 expression plasmid (TPT1) co-transfected with miR-216b-5p expression plasmid. Then, CCK-8 assays and flow cytometry, were used to determine cell proliferation (C), cell cycle (D), and cell apoptosis (E), respectively. The experiments were independently repeated three times. *, $P<0.05 ; * *, P<0.01$.

\section{The molecular mechanism of miR-216b-5p influencing pancreatic cancer cell progression by targeting TPT 1}

In pancreatic cancer, TPT1 interacts with Pim-3 and enhances Pim-3 protein stability, thereby regulating cell proliferation, cell cycle and apoptosis [26]. In this study, we found that miR-216b-5p could regulate pancreatic cancer cell progression by targeting TPT1. Therefore, miR-216b-5p may inhibit pancreatic cancer cell progression by regulating the Pim-3 signaling. To determine whether miR-216b-5p regulates Pim-3 signaling, we examined TPT1, Pim-3, Cyclin B1, p-Bad (Ser112), Bad and Bcl-xL by Western blot. We found that miR-216b-5p up-regulation in Bxpc-3 and SW1990 cells significantly inhibited TPT1, Pim-3, Cyclin B1, p-Bad (Ser112) and Bcl-xL protein expression (Figure 6A). In addition, to further determine whether miR-216b-5p could regulate Pim-3 signaling by the inhibition of TPT1, we up-regulated TPT1 expression in Bxpc-3 and SW1990 cells after transfecting the miR-216b-5p expression plasmid. Our results showed that the ectopic expression of TPT1 reversed the inhibition of TPT1, Pim-3, Cyclin B1, p-Bad (Ser112) and Bcl-xL protein expression caused by miR-216b-5p over-expression (Figure 6B). Taken together, miR-216b-5p may affect pancreatic cancer progression via TPT1/Pim-3 signaling (Figure 7).

\section{Discussion}

Recently, miRNAs have been shown to be important in cancer initiation and tumor progression [30]. Among these functional miRNAs, miR-216b is significantly down-regulated in many cancer types and mostly acts as a tumor suppressor [14-17]. In addition, miR-216b expression was also decreased in pancreatic cancer tissues and associated with aggressive tumor characteristics and shortened disease-free survival [18]. Accordingly, we found that miR-216b-5p was significantly decreased in pancreatic cancer tissues and cell lines (Figure $1 \mathrm{~A}$ and $\mathrm{C}$ ). Meanwhile, low miR-216b-5p expression was significantly associated with large tumor size, advanced TNM stage and shorter survival. Azevedo-Pouly AC et al. [27] found that miR-216b expression was decreased with age (and phenotype severity) in $\mathrm{P} 48^{+/ C r e}$ and LSL-KRASG12D transgenic mouse models of pancreatic adenocarcinoma. Thus, it is necessary to study the roles and the molecular mechanisms of miR-216b-5p in pancreatic cancer progression. 
A

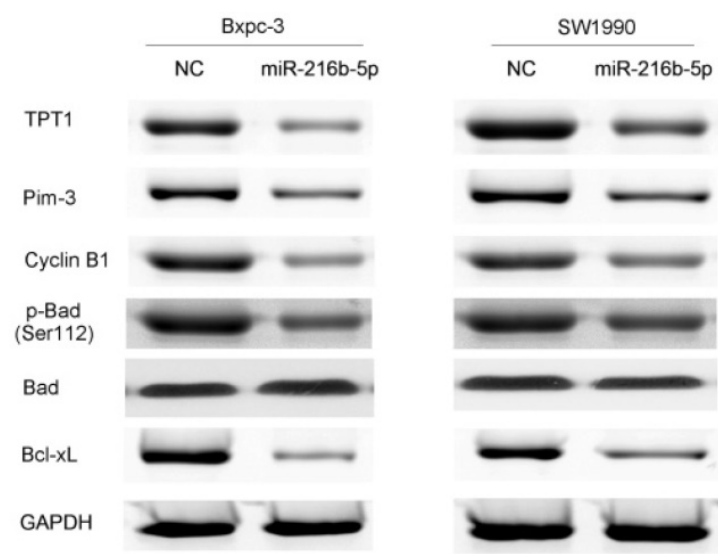

B

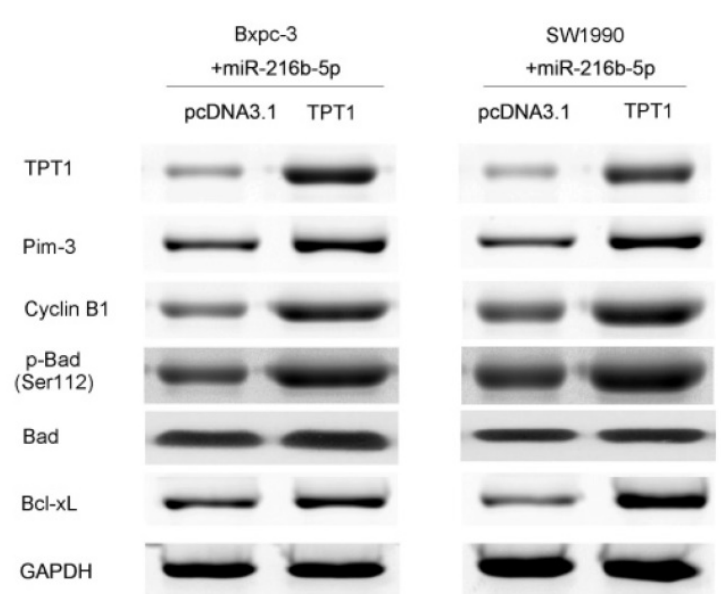

Figure 6. miR-216b-5p inhibites pancreatic cancer cell progression by regulating TPT1/Pim-3 signaling. (A) Bxpc-3 and SW1990 cells were transfected with the scrambled miRNA expression plasmid (NC) or miR-216b-5p expression plasmid. The expression levels of TPT1, Pim-3, p-Bad (Ser112), Bad and Bcl-xL protein were analyzed by Western blot. (D) Bxpc-3 and SW1990 cells were transfected with pcDNA3.1 or TPT1 expression plasmid (TPTI) co-transfected with miR-216b-5p expression plasmid. The expression levels of TPT1, Pim-3, p-Bad (Ser112), Bad and Bcl-xL protein were analyzed by Western blot. GAPDH was used as a loading control.

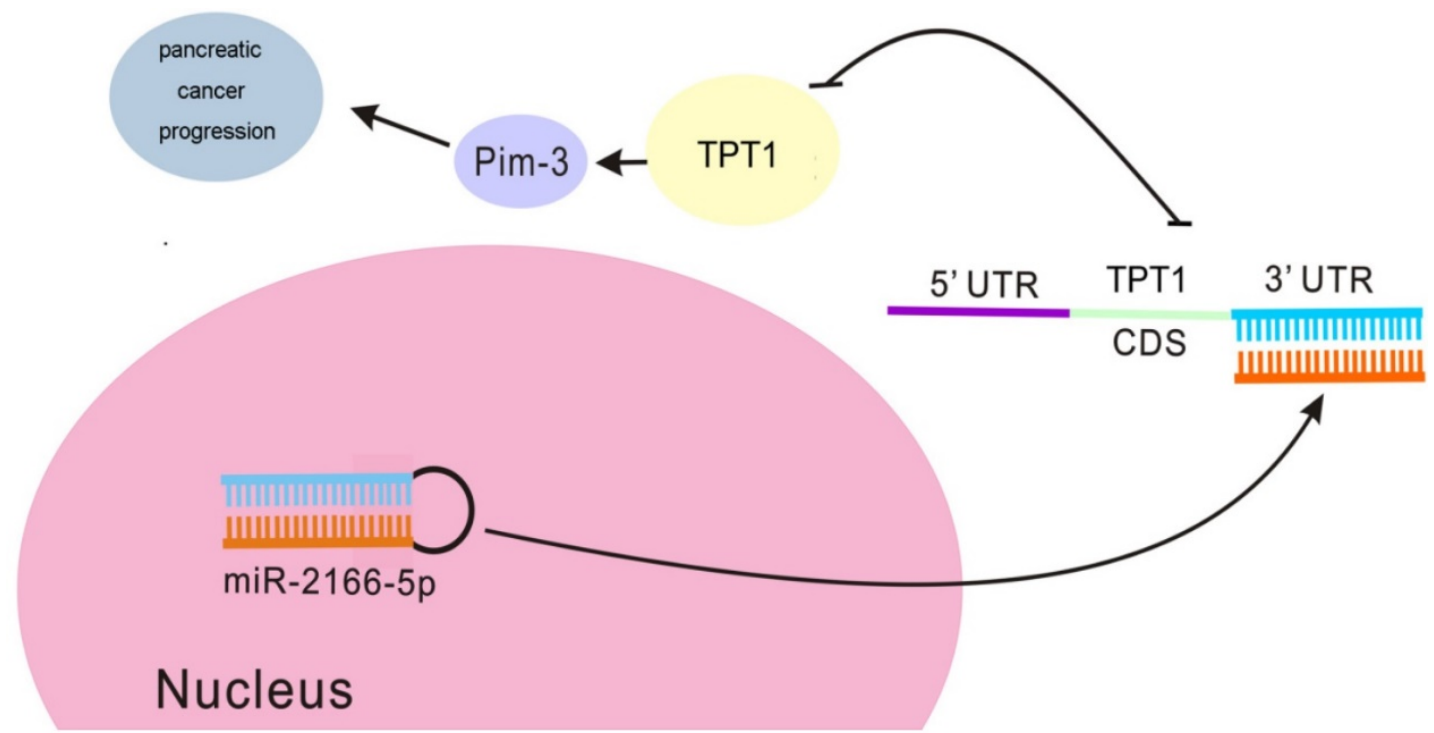

Figure 7. The suggested molecular mechanism of the antitumor effect of miR-216b-5p in pancreatic cancer. miR-216b-5p up-regulation inhibits TPT1 expression and subsequently suppresses the activation of Pim-3 signaling, thereby regulating cell proliferation, cell cycle and apoptosis.

In the present study, our results showed that the transfection of pancreatic cancer cells with miR-216b-5p expression plasmid inhibited cell proliferation (Figure 2B). Next, to determine whether the suppression of cell proliferation was due to the inhibition of cell cycle or the promotion of apoptosis by miR-216b-5p, we carried out cell cycle and apoptosis assays in vitro. Our results indicated that miR-216b-5p up-regulation induced cell cycle arrest and apoptosis (Figure 2C and D) and repressed tumor growth in vivo ((Figure 2E). Interestingly, Deng $\mathrm{M}$ et al. [14] found that miR-216b could suppress cell proliferation in nasopharyngeal carcinoma. In addition, Wang Y et al. [17] found that miR-216b up-regulation inhibits proliferation and cell cycle progression in human gastric adenocarcinoma cells.
In U2OS cells, miR-216b suppresses c-Jun expression, thereby triggering cell death [28]. Therefore, these results suggest that targeting miR-216b-5p could be a promising therapeutic measure in pancreatic cancer patients.

It is well known that miRNAs can potentially affect various physiological and pathological processes by directly regulating target gene expression. Recently, it has been reported that miR-216b-5p targets many important genes such as KRAS, IGF2BP2, HDAC8, FGFR1, c-Jun [14-17, 28]. To study the underlying mechanism of miR-216b-5p in pancreatic cancer, we next explored the possible target genes of miR-216b-5p using bioinformatics tools. Two putative targets, TPT1 and SOX4, attracted our attention immediately. SOX4 expression in PDAC 
correlates with shorter survival [29]. Moreover, SOX4 suppression reduced cell proliferation in pancreatic cancer cell lines [29]. In pancreatic cancer, TPT1 functions as an oncogene and contributes to pancreatic cancer development and progression [26]. In the present study, our results showed that TPT1 protein expression was significantly suppressed in cells after transfection with the miR-216b-5p expression plasmid, while SOX4 protein expression was not changed (Figure S1). Further investigation showed that TPT1 was a direct functional target of miR-216b-5p in pancreatic cancer. First, ectopic miR-216b-5p expression significantly inhibited the activity of the luciferase reporter vector containing the TPT1 3'UTR (Figure 3D). Second, the miR-216b-5p-mediated inhibition of luciferase activity was reversed by the mutated binding site (Figure 3D). Third, TPT1 overexpression was frequently observed in pancreatic cancer tissues compared with cancer-adjacent normal tissues, and an inverse correlation was found between the expression of miR-216b-5p and TPT1 in pancreatic cancer tissues (Figure 3E and F). Moreover, TPT1 down-regulation suppressed pancreatic cancer cell proliferation and cell cycle progression and induced cell apoptosis (Figure 4). In addition, TPT1 restoration partially reversed the effect of miR-216b-5p up-regulation on pancreatic cancer cell proliferation, cell cycle progression and apoptosis (Figure 5). These results suggested that the regulation of TPT1 expression by miR-216b-5p is partly responsible for pancreatic cancer progression.

In this study, we investigated the regulatory relationship between miR-216b-5p and TPT1, but the downstream mechanisms underlying the antitumor effect of miR-216b-5p-TPT1 axis were poorly explored. Pim-3, a member of the proto-oncogene Pim family that expresses serine/threonine kinase activity, could regulate cell proliferation, cell cycle and cell survival in carcinogenesis [26, 30-34]. In pancreatic cancer, Pim-3 may prevent apoptosis by regulating the phosphorylation of Bad and the expression of an antiapoptotic molecule, Bcl-xL [35]. In addition, TPT1 could interact with Pim-3 and enhance Pim-3 protein stability by blocking the ubiquitin-proteasomemediated degradation of the Pim-3 protein, thereby promoting tumor growth in pancreatic cancer. In the present study, we confirmed the regulatory relationship between miR-216b-5p, a known tumor-suppressive miRNA, and an oncogene, TPT1, and hypothesized that miR-216b-5p executes a tumor-suppressive function by inducing Pim-3 activation. Therefore, further studies are required to explore the effect of miR-216b-5p on Pim-3 signaling. Herein, we found that miR-216b-5p overexpression in
Bxpc-3 and SW1990 cells significantly inhibited TPT1, Pim-3, p-Bad (Ser112) and Bcl-xL protein expression (Figure 6A). In addition, we found that TPT1 restoration reversed the inhibition of TPT1, Pim-3, p-Bad (Ser112) and Bcl-xL protein expression caused by miR-216b-5p over-expression (Figure 6D). Thus, miR-216b-5p may affect Pim-3 signaling by TPT1 and regulate pancreatic cancer progression via TPT1/Pim-3 signaling (Figure 7).

In summary, our results indicated that miR-216b-5p was down-regulated in pancreatic cancer and functioned as a potential tumor-suppressor. We identified TPT1 as a novel target of miR-216b-5p. We also demonstrated that miR-216b-5p suppressed cell proliferation and induced cell cycle arrest and apoptosis via the TPT1/Pim-3 axis in pancreatic cancer. These findings suggest that miR-216b-5p may be a new potential therapeutic target in pancreatic cancer.

\section{Supplementary Material}

Figure S1. http://www.jcancer.org/v08p2854s1.pdf

\section{Acknowledgments}

This study was supported by the National Natural Science Foundation of China (Grant No.81472288).

\section{Competing Interests}

The authors have declared that no competing interest exists.

\section{References}

1. Karanikas M, Esempidis A, Chasan ZT, Deftereou T, Antonopoulou M, Bozali F, et al. Pancreatic Cancer from Molecular Pathways to Treatment Opinion. J Cancer. 2016; 7: 1328-39.

2. Akinleye A, Iragavarapu C, Furqan M, Cang S, Liu D. Novel agents for advanced pancreatic cancer. Oncotarget. 2015; 6: 39521-37.

3. Rachagani S, Macha MA, Heimann N, Seshacharyulu P, Haridas D, Chugh S, et al. Clinical implications of miRNAs in the pathogenesis, diagnosis and therapy of pancreatic cancer. Adv Drug Deliv Rev. 2015; 81:16-33.

4. Bartel DP. MicroRNAs: genomics, biogenesis, mechanism, and function. Cell. 2004; 116: 281-97.

5. Tay Y, Zhang J, Thomson AM, Lim B, Rigoutsos I. MicroRNAs to Nanog, Oct4 and Sox2 coding regions modulate embryonic stem cell differentiation. Nature. 2008; 455: 1124-8.

6. Huntzinger E., Izaurralde E. Gene silencing by microRNAs: contributions of translational repression and mRNA decay. Nat Rev Genet. 2011; 12: 99-110.

7. Stahlhut C, Slack FJ. MicroRNAs and the cancer phenotype: profiling, signatures and clinical implications. Genome Med. 2013; 5:111.

8. Chen PS, Su JL, Hung MC. Dysregulation of microRNAs in cancer. J Biomed Sci. 2012; 19: 90.

9. Iorio MV Croce CM. microRNA involvement in human cancer. Carcinogenesis. 2012; 33:1126-33.

10. Brunetti O, Russo A, Scarpa A, Santini D, Reni M, Bittoni A, et al. MicroRNA in pancreatic adenocarcinoma: predictive/prognostic biomarkers or therapeutic targets? Oncotarget. 2015; 6: 23323-41.

11. Moriyama T, Ohuchida K, Mizumoto K, Yu J, Sato N, Nabae T, et al. MicroRNA-21 modulates biological functions of pancreatic cancer cells including their proliferation, invasion, and chemoresistance. Mol Cancer Ther. 2009; 8: 1067-74.

12. Dong J, Zhao YP, Zhou L, Zhang TP, Chen G. Bcl-2 upregulation induced by miR-21 via a direct interaction is associated with apoptosis and chemoresistance in MIA PaCa-2 pancreatic cancer cells. Arch Med Res. 2011; 42: 8-14. 
13. Gao W, Gu Y, Li Z, Cai H, Peng Q, Tu M, et al. MiR-615-5p is epigenetically inactivated and functions as a tumor suppressor in pancreatic ductal adenocarcinoma. Oncogene. 2014; 34: 1629-40.

14. Deng M, Tang H, Zhou $\mathrm{Y}$, Zhou M, Xiong W, Zheng $\mathrm{Y}$, et al. miR-216b suppresses tumor growth and invasion by targeting KRAS in nasopharyngeal carcinoma. J Cell Sci. 2011; 124:2997-3005.

15. Wang F, Ying HQ, He BS, Pan YQ, Deng OW, Sun HL,et al. Upregulated lncRNA-UCA1 contributes to progression of hepatocellular carcinoma through inhibition of miR-216b and activation of FGFR1/ERK signaling pathway. Oncotarget. 2015; 6: 7899-917.

16. Liu FY, Zhou SJ, Deng YL, Zhang ZY, Zhang EL, Wu ZB, et al. MiR-216b is involved in pathogenesis and progression of hepatocellular carcinoma through HBx-miR-216b-IGF2BP2 signaling pathway. Cell Death Dis. 2015; 6:e1670.

17. Wang Y, Xu P, Yao J, Yang R, Shi Z, Zhu X, et al. MicroRNA-216b is Down-Regulated in Human Gastric Adenocarcinoma and Inhibits Proliferation and Cell Cycle Progression by Targeting Oncogene HDAC8. Target Oncol. 2016; 11: 197-207.

18. Egeli U, Tezcan G, Cecener G, Tunca B, Demirdogen Sevinc E, Kaya E, et al. miR-216b Targets FGFR1 and Confers Sensitivity to Radiotherapy in Pancreatic Ductal Adenocarcinoma Patients Without EGFR or KRAS Mutation. Pancreas. 2016; 45: 1294-302.

19. Chan T H M, Chen L, Guan X Y. Role of Translationally Controlled Tumor Protein in Cancer Progression. Biochem Res Int. 2012; 2012: 369384.

20. Koziol MJ, Gurdon JB. TCTP in development and cancer. Biochem Res Int. 2012; 2012: 105203

21. Wu D, Guo Z, Min W, Zhou B, Li M, Li W, et al. Upregulation of TCTP expression in human skin squamous cell carcinoma increases tumor cell viability through anti-apoptotic action of the protein. Exp Ther Med. 2012; 3: 437-42.

22. Gu X, Yao L, Ma G, Cui L, Li Y, Liang W, et al. TCTP promotes glioma cell proliferation in vitro and in vivo via enhanced $\beta$-catenin/TCF-4 transcription. Neuro Oncol. 2014; 16: 217-27.

23. Kaarbø M, Storm ML, Qu S, Wæhre H, Risberg B, Danielsen HE, et al. TCTP is an androgen-regulated gene implicated in prostate cancer. PLoS One. 2013; 8: e69398.

24. Gnanasekar M, Thirugnanam S, Zheng G, Chen A, Ramaswamy K. Gene silencing of translationally controlled tumor protein (TCTP) by siRNA inhibits cell growth and induces apoptosis of human prostate cancer cells. Int J Oncol. 2009; 34: 1241-6.

25. Jung J, Kim HY, Kim M, Sohn K, Kim M, Lee K. Translationally controlled tumor protein induces human breast epithelial cell transformation through the activation of Src. Oncogene. 2011; 30: 2264-74.

26. Zhang F, Liu B, Wang Z, Yu XJ, Ni QX, Yang WT, et al. A novel regulatory mechanism of Pim-3 kinase stability and its involvement in pancreatic cancer progression. Mol Cancer Res. 2013; 11:1508-20.

27. Azevedo-Pouly AC, Sutaria DS, Jiang J, Elgamal OA, Amari F, Allard D, et al. miR-216 and miR-217 expression is reduced in transgenic mouse models of pancreatic adenocarcinoma, knockout of miR-216/miR-217 host gene is embryonic lethal. Funct Integr Genomics. 2016; 19: 1-10.

$28 \mathrm{Xu}$ Z, Bu Y, Chitnis N, Koumenis C, Fuchs SY, Diehl JA. miR-216b regulation of c-Jun mediates GADD153/CHOP-dependent apoptosis. Nat Commun. 2016; 7: 11422

29. Huang HY, Cheng YY, Liao WC, Tien YW, Yang CH, Hsu SM, et al. SOX4 transcriptionally regulates multiple SEMA3/plexin family members and promotes tumor growth in pancreatic cancer. PLoS One. 2012; 7: e48637.

30. Mukaida N, Wang YY, Li YY. Roles of Pim-3, a novel survival kinase, in tumorigenesis. Cancer Sci. 2011; 102: 1437-42.

31. Popivanova BK, Li YY, Zheng H, Omura K, Fujii C, Tsuneyama K, Mukaida N. Proto-oncogene, Pim-3 with serine/threonine kinase activity, is aberrantly expressed in human colon cancer cells and can prevent Bad-mediated apoptosis. Cancer Sci. 2007; 98:321-8.

32. Fujii C, Nakamoto Y, Lu P, Tsuneyama K, Popivanova BK, Kaneko S, Mukaida N. Aberrant expression of serine/threonine kinase Pim-3 in hepatocellular carcinoma development and its role in the proliferation of human hepatoma cell lines. Int J Cancer. 2005; 114: 209-18.

33. Li YY, Mukaida N. Pathophysiological roles of Pim-3 kinase in pancreatic cancer development and progression. World J Gastroenterol. 2014; 20: 9392-404.

34. Mukaida N, Wang YY, Li YY. Roles of Pim-3, a novel survival kinase, in tumorigenesis. Cancer Sci. 2011; 102: 1437-42.

35. Li YY, Popivanova BK, Nagai $Y$, Ishikura H, Fujii C, Mukaida N. Pim-3, a proto-oncogene with serine/threonine kinase activity, is aberrantly expressed in human pancreatic cancer and phosphorylates bad to block bad-mediated apoptosis in human pancreatic cancer cell lines. Cancer Res. 2006; 66: 6741-7. 